\title{
1 WEAKLY HYPERBOLIC EQUATIONS - A MODERN FIELD IN THE THEORY OF HYPERBOLIC EQUATIONS
}

M. Dreher and M. Reissig Faculty for Mathematics and Computer Sciences Freiberg University of Mining and Technology Bernhard-von-Cotta-Str. 2

09596 Freiberg, Germany (dreher,reissig)@mathe.tu-freiberg.de

\subsection{INTRODUCTION}

In this paper we would like to give an overview of problems and new trends in the theory of weakly hyperbolic equations.

We start with the Cauchy problem

$$
u_{t t}-a(t) u_{x x}=0, \quad u(x, 0)=u_{0}(x), \quad u_{t}(x, 0)=u_{1}(x) .
$$

In the strictly hyperbolic theory $(a(t) \geq c>0)$ we have the correctness in the classes of Sobolev spaces and the estimate

$$
\begin{aligned}
\sup _{[0, T]}\left(\|u(t)\|_{H^{m+1-\beta t}}+\right. & \left.\left\|u^{\prime}(t)\right\|_{H^{m-\beta t}}\right) \\
& \leq C_{0}\left(1+e^{C_{m} T}\right)\left(\left\|u_{0}\right\|_{H^{m+1}}+\left\|u_{1}\right\|_{H^{m}}\right)
\end{aligned}
$$


for a $\log$-lipschitz coefficient $a(t)$, see [CL95].

In the weakly hyperbolic theory $(a(t) \geq 0)$ the situation is completely different. $C^{\infty}$-regularity of $a(t)$ is not sufficient for well-posedness of the Cauchy problem even in the classes of distributions. In [CS82] it was shown that for any $T>0$ a coefficient $a(t) \in C^{\infty}([0, \infty))$ and $C^{\infty}$ data $u_{0}, u_{1}$ exist, such that the solution belongs to $C^{\infty}\left([0, T), C^{\infty}(\mathbb{R})\right)$, but not to $C\left([0, T], \mathcal{D}^{\prime}(\mathbb{R})\right)$. This function $a(t)$ is positive for $t<T$, oscillating for $t \rightarrow T-0$ and identically zero for $t \geq T$.

We list three ways to restrict the influence of oscillations:

- One assumes a nonlocal condition of the form

$$
\int_{0}^{T} \frac{\left|a^{\prime}(\tau)\right|}{a(\tau)+\varepsilon} d \tau \leq C|\ln \varepsilon|,
$$

see [CJS83].

- The coefficient $a(t)$ is assumed to be analytic, see [D'A95].

- One supposes $a=a(x, t)=\sigma^{2}(x) \lambda^{2}(t)$ with $\lambda(0)=0, \lambda^{\prime}(t)>0$, see $[\mathrm{Ole} 70]$.

In opposite to the strictly hyperbolic case, lower order terms have an influ-

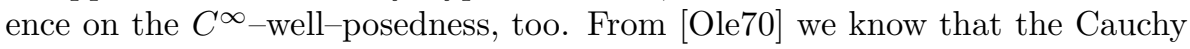
problem for

$$
u_{t t}-t^{2 l} x^{2 n} u_{x x}+t^{k} x^{m} u_{x}=0
$$

is $C^{\infty}$ - well-posed if $k \geq l-1, m \geq n$. The necessity of this conditions was proved in [IP74]. These conditions are called Levi conditions of $C^{\infty}$-type which are used to ensure the well-posedness in $C^{\infty}$. This example shows that one has to distinguish between Levi conditions for time degeneracy and Levi conditions for spatial degeneracy.

One possibility to formulate Levi conditions of $C^{\infty}$-type for the equation

$$
u_{t t}-\sigma^{2}(x) \lambda^{2}(t) u_{x x}+b(x, t) u_{x}+c(x, t) u_{t}+d(x, t) u=g(x, t)
$$

is to assume

$$
|b(x, t)| \leq C|\sigma(x)| \lambda^{\prime}(t),
$$

compare with $\sigma(x)=x^{n}, \lambda(t)=t^{l}, b(x, t)=t^{k} x^{m}$. Now let us turn to quasilinear problems, e.g.

$$
u_{t t}-\sigma^{2}(x) \lambda^{2}(t) u_{x x}=f\left(x, t, u, u_{t}, u_{x}\right) .
$$

In this case we have the Levi condition of $C^{\infty}$-type

$$
\left|\frac{\partial f}{\partial p}(x, t, u, v, p)\right| \leq C|\sigma(x)| \lambda^{\prime}(t) .
$$


In the case of weakly hyperbolic equations another phenomenon occurs - the loss of Sobolev regularity, even if the coefficients are smooth and have no oscillations. Let us consider the Cauchy problem

$$
u_{t t}-t^{2} u_{x x}=a u_{x}, \quad u(x, 0)=\varphi(x), \quad u_{t}(x, 0)=0 .
$$

As it was shown in [Qi 58], the solution can be represented in the form

$$
u(x, t)=\sum_{k=0}^{n} c_{k} t^{2 k} \partial_{x}^{k} \varphi\left(x+\frac{t^{2}}{2}\right)
$$

if $a=4 n+1, n \in \mathbb{N}_{0}$. In other words, if $\varphi \in H^{s}$, then $u(., t) \in H^{s-\frac{a-1}{4}}$.

Typical problems for the linear equation (1.1) are:

- well-posedness of the Cauchy problem in Sobolev spaces, $C^{\infty}$ or Gevrey spaces; well-posedness means existence and cone of dependence as usual;

- propagation of singularities;

- construction of parametrix;

- influence of oscillations in the coefficients.

Typical problems for the quasilinear equation (1.2) are:

- local existence of solutions in Sobolev spaces, $C^{\infty}$ or Gevrey spaces;

- uniqueness of solutions of the Cauchy problem and existence of a cone of dependence;

- global regularity of solutions of the Cauchy problem;

- stability of solutions.

A natural way to attack the problem to prove the existence of Sobolev solutions for the Cauchy problem for (1.2) is to linearize the equation and to apply fixed points arguments. A so-called strictly hyperbolic type estimate for the linearized equation is essential for this purpose. That means:

It exists a Banach space $B$ such that the following holds:

If $\frac{f(x, t)}{\lambda^{\prime}(t)} \in B$, then the solution of

$$
u_{t t}-\sigma^{2}(x) \lambda^{2}(t) u_{x x}=f(x, t), \quad u(x, 0)=u_{t}(x, 0)=0
$$

satisfies $\frac{u}{\lambda}, \frac{u_{t}}{\lambda}, \sigma u_{x} \in B$ with

$$
\left\|\frac{u}{\lambda}\right\|_{B}+\left\|\frac{u_{t}}{\lambda}\right\|_{B}+\left\|\sigma u_{x}\right\|_{B} \leq C\|f\|_{B} .
$$

In the strictly hyperbolic case this property holds with $B=C\left([0, T], H^{s}\right)$, $\sigma(x)=1$ and $\lambda(t)=1+t$.

If such a strictly hyperbolic type estimate holds for the linear problem, then one can mostly construct a sequence of approximate solutions of (1.2) in the usual way which will converge for small $t$ to a solution.

In the case of weakly hyperbolic equations it is not obvious how to derive such an estimate and how to fix the space $B$, since we have to expect a loss of Sobolev regularity. 


\subsection{WEAKLY HYPERBOLIC EQUATIONS WITH TIME AND SPATIAL DEGENERACY, BUT WITHOUT OSCILLATIONS}

In this section we consider the model problem with time and spatial degeneracy, but without oscillations of the coefficients of the main part

$$
\begin{aligned}
& u_{t t}-\sum_{i=1}^{n} \partial_{x_{i}}\left(\lambda^{2}(t) \sigma^{2}(x) u_{x_{i}}(x, t)\right)=f\left(b_{1}(x, t) u_{x_{1}}(x, t), \ldots, b_{n}(x, t) u_{x_{n}}(x, t)\right), \\
& u(x, 0)=\varphi_{0}(x), \quad u_{t}(x, 0)=\varphi_{1}(x)
\end{aligned}
$$

under the following assumptions:

$$
\begin{aligned}
& \sigma \in C_{b}^{\infty}\left(\mathbb{R}^{n}\right), \quad \varphi_{0} \in H^{N+1}\left(\mathbb{R}^{n}\right), \quad \varphi_{1} \in H^{N}\left(\mathbb{R}^{n}\right), \\
& \exists M>0: \quad \operatorname{supp}\left(\left|\varphi_{0}\right|+\left|\varphi_{1}\right|\right) \subset[-M, M], \\
& b_{i} \in C\left(\left[0, T_{0}\right], C_{b}^{\infty}\left(\mathbb{R}^{n}\right)\right), \quad f \in C^{\infty}\left(\mathbb{R}^{n}\right), \\
& \lambda \in C^{1}\left(\left[0, T_{0}\right]\right), \quad \lambda(0)=\lambda^{\prime}(0)=0, \quad \lambda^{\prime}(t)>0 \quad(t>0), \\
& \left|b_{i}(x, t)\right| \leq C_{b}|\sigma(x)| \lambda^{\prime}(t), \quad(x, t) \in \mathbb{R}^{n} \times\left[0, T_{0}\right], \quad i=1, \ldots, n, \\
& \left|\frac{\partial f}{\partial p_{i}}\left(p_{1}, \ldots, p_{n}\right)\right| \leq C_{f}, \quad\left|p_{1}\right|+\cdots+\left|p_{n}\right| \leq \delta, \quad i=1, \ldots, n, \\
& f(0)=0 .
\end{aligned}
$$

The functions $\sigma$ and $\lambda$ are used to formulate the degeneration, which occurs at the points $\left(x_{0}, t_{0}\right)$ with $\sigma\left(x_{0}\right)=0$ or $\lambda\left(t_{0}\right)=0$. The assumption (1.8) describes the Levi condition and the assumption (1.7) excludes oscillations with respect to $t$ in the coefficients.

We want to prove the local existence of a solution in Sobolev spaces and the finite speed of propagation of this solution. As one can see from the example of Qi Min-You, we have to expect a loss of regularity, i.e. the solution will belong to a space $C^{1}\left([0, T], H^{N-\gamma}\right)$. The number $\gamma$ of lost derivatives will be proportional to the product of the constants $C_{b}, C_{f}$ from (1.8), (1.9). The assumption $f(0)=0$ will only be used in the proof of the finite speed of propagation.

Our approach will be divided into the following steps:

- reduction of the starting problem (1.3) to a family of Cauchy problems for ordinary differential equations and for a quasilinear weakly hyperbolic equation whose right-hand side has a suitable asymptotic behaviour (in this step we feel the loss of derivatives);

- derivation of a strictly hyperbolic type property for the solutions of the linearized weakly hyperbolic Cauchy problems by using the energy method, a Gronwall type lemma for differential inequalities with a singular coefficient and an approximation argument;

- using the results from the previous step, a fixed point argument gives a solution of the auxiliary problem, starting problem, respectively. 
a) We write

$$
u(x, t)=u^{0}(x, t)+\cdots+u^{d}(x, t)+v(x, t),
$$

where the functions $u^{1}, \ldots, u^{n}$ are solutions of ordinary differential equations in $t$ with the parameter $x$ :

$$
\begin{aligned}
& u_{t t}^{0}=f(0), \quad u^{0}(x, 0)=\varphi_{0}(x), \quad u_{t}^{0}(x, 0)=\varphi_{1}(x), \\
& \ldots, \\
& u_{t t}^{j}=f\left(b_{1} \sum_{l=0}^{j-1} u_{x_{1}}^{l}, \ldots, b_{n} \sum_{l=0}^{j-1} u_{x_{n}}^{l}\right)-f\left(b_{1} \sum_{l=0}^{j-2} u_{x_{1}}^{l}, \ldots, b_{n} \sum_{l=0}^{j-2} u_{x_{n}}^{l}\right) \\
& \quad+\sum_{i=1}^{n} \partial_{x_{i}}\left(\lambda^{2} \sigma^{2} u_{x_{i}}^{j-1}\right), \\
& u^{j}(x, 0)=u_{t}^{j}(x, 0)=0, \quad j=1, \ldots, d, \quad \sum_{l=0}^{-1}=0 .
\end{aligned}
$$

This system can be solved step by step, we get solutions

$$
u^{j} \in C^{2}\left(\left[0, T_{j}\right], H^{N-2 j}\right) .
$$

By Hadamard's formula and (1.8) we see that

$$
\left\|u^{j}(t)\right\|_{H^{N-2 j}}+\left\|u_{t}^{j}(t)\right\|_{H^{N-2 j}} \leq C \lambda^{j}(t)
$$

The function $u$ is a solution of (1.3), if and only if $v$ solves the Cauchy problem

$$
\begin{aligned}
v_{t t}-\sum_{i=1}^{n} \partial_{x_{i}}\left(\lambda^{2} \sigma^{2} v_{x_{i}}\right) & =f\left(b_{1}\left(\sum_{l=0}^{d} u_{x_{1}}^{l}+v_{x_{1}}\right), \ldots, b_{n}\left(\sum_{l=0}^{d} u_{x_{n}}^{l}+v_{x_{n}}\right)\right) \\
& -f\left(b_{1} \sum_{l=0}^{d-1} u_{x_{1}}^{l}, \ldots, b_{n} \sum_{l=0}^{d-1} u_{x_{n}}^{l}\right)+\sum_{i=1}^{n} \partial_{x_{i}}\left(\lambda^{2} \sigma^{2} u_{x_{i}}^{d}\right) \\
= & : f_{d}\left(x, t, b_{1} v_{x_{1}}, \ldots, b_{n} v_{x_{n}}\right), \\
v(x, 0)=v_{t}(x, 0)=0 . &
\end{aligned}
$$

The right-hand side satisfies

$$
\begin{aligned}
& \left\|f_{d}(., t, 0)\right\|_{H^{N-2 d-2}} \leq C_{d} \lambda^{d}(t) \lambda^{\prime}(t) \\
& \left\|\frac{\partial f_{d}}{\partial p_{i}}\left(., t, p_{1}(., t), \ldots, p_{n}(., t)\right)\right\|_{\infty} \leq C_{f}, \quad\left|p_{1}\right|+\cdots+\left|p_{n}\right| \leq \delta^{\prime}
\end{aligned}
$$

for small $t$. We write $N_{0}:=N-2 d-2$ and from Hadamard's formula we 
deduce that

$$
\begin{aligned}
\| f_{d}(x, t, & \left.b_{1} v_{x_{1}}, \ldots, b_{n} v_{x_{n}}\right) \|_{H^{N_{0}}} \\
& \leq\left\|f_{d}\left(x, t, b_{1} v_{x_{1}}, \ldots, b_{n} v_{x_{n}}\right)-f_{d}(., t, 0)\right\|_{H^{N_{0}}}+\left\|f_{d}(., t, 0)\right\|_{H^{N_{0}}} \\
& \leq C_{f} \sum_{i=1}^{n} \sum_{|\alpha|=N_{0}}\left\|\partial_{x}^{\alpha}\left(b_{i} v_{x_{i}}\right)\right\|_{2}+C \lambda^{\prime}(t)\|v\|_{H^{N_{0}}}+C_{d} \lambda^{d}(t) \lambda^{\prime}(t) \\
& \leq C_{f} \sum_{i=1}^{n} \sum_{|\alpha|=N_{0}}\left\|b_{i} \partial_{x}^{\alpha} v_{x_{i}}\right\|_{2}+C_{d}^{\prime} \lambda^{\prime}(t)\|v\|_{H^{N_{0}}}+C_{d} \lambda^{d}(t) \lambda^{\prime}(t)
\end{aligned}
$$

for $\sum_{i=1}^{n}\left|b_{i}\right|\left(\sum_{l=0}^{d}\left|u_{x_{i}}^{l}\right|+\left|v_{x_{i}}\right|\right) \leq \delta$. With $a(x, t):=\sigma^{2}(x) \lambda^{2}(t)$ we define the energies

$$
\begin{aligned}
& e_{j}(v)(t)^{2}=\sum_{|\alpha|=j} \int a(x, t) \sum_{i=1}^{n}\left(\partial_{x}^{\alpha} \partial_{x_{i}} v\right)^{2}+\left(\partial_{x}^{\alpha} v_{t}\right)^{2}+\left(\partial_{x}^{\alpha} v\right)^{2} d x, \\
& E_{N}(v)(t)=\sum_{j=0}^{N} e_{j}(v)(t)
\end{aligned}
$$

and obtain

$$
\begin{aligned}
\left\|f_{d}\left(., t, b_{1} v_{x_{1}}, \ldots, b_{n} v_{x_{n}}\right)\right\|_{H^{N_{0}}} \leq & \left(\sqrt{n} C_{f} C_{b} \frac{\lambda^{\prime}(t)}{\lambda(t)}+C_{d}^{\prime} \lambda^{\prime}(t)\right) E_{N_{0}}(v)(t) \\
& +C_{d} \lambda^{d}(t) \lambda^{\prime}(t) .
\end{aligned}
$$

b) For further considerations we need an estimate for solutions of

$$
\begin{aligned}
& w_{t t}-\sum_{i=1}^{n} \partial_{x_{i}}\left(\lambda^{2} \sigma^{2} w_{x_{i}}\right)=g(x, t), \quad w(x, 0)=w_{t}(x, 0)=0, \\
& \|g(t)\|_{H^{N_{0}}} \leq C_{g} \lambda^{d-1}(t) \lambda^{\prime}(t)+C_{g}^{\prime} \lambda^{d}(t) \lambda^{\prime}(t) .
\end{aligned}
$$

We suppose that the function $g \in C\left(\left[0, T_{0}\right], H^{N_{0}}\right)$ has compact support with respect to $x$ and that there exists a solution $w$ with compact support with respect to $x$ which satisfies

$$
w, w_{t}, \sigma w_{x} \in C\left(\left[0, T_{0}\right], H^{N_{0}}\right) .
$$

Differentiating $e_{j}(w)(t)^{2}$ and using $a_{t}=2 \frac{\lambda^{\prime}(t)}{\lambda(t)} a$ and the differential equation we get

$$
\begin{gathered}
e_{j}(w)(t) e_{j}(w)^{\prime}(t)=\sum_{|\alpha|=j} \int \frac{\lambda^{\prime}(t)}{\lambda(t)} a \sum_{i=1}^{n}\left(\partial_{x}^{\alpha} \partial_{x_{i}} w\right)^{2}+a \sum_{i=1}^{n}\left(\partial_{x}^{\alpha} \partial_{x_{i}} w\right)\left(\partial_{x}^{\alpha} \partial_{x_{i}} w_{t}\right) \\
+\left(\partial_{x}^{\alpha} w_{t}\right)\left(\partial_{x}^{\alpha} g\right)+\left(\partial_{x}^{\alpha} w_{t}\right) \sum_{i=1}^{n} \partial_{x}^{\alpha} \partial_{x_{i}}\left(a w_{x_{i}}\right)+\left(\partial_{x}^{\alpha} w\right)\left(\partial_{x}^{\alpha} w_{t}\right) d x
\end{gathered}
$$


By the aid of partial integration and the Leibniz formula we get

$$
\begin{aligned}
& \int\left(\partial_{x}^{\alpha} w_{t}\right) \sum_{i=1}^{n} \partial_{x}^{\alpha} \partial_{x_{i}}\left(a w_{x_{i}}\right) d x \\
& =\int \sum_{\substack{\beta \leq \alpha \\
\beta \neq 0}}\left(\begin{array}{l}
\alpha \\
\beta
\end{array}\right)\left(\partial_{x}^{\alpha} w_{t}\right) \sum_{i=1}^{n} \partial_{x_{i}}\left(\left(\partial_{x}^{\beta} a\right)\left(\partial_{x}^{\alpha-\beta} w_{x_{i}}\right)\right)-\left(\partial_{x}^{\alpha} \partial_{x_{i}} w_{t}\right) a \sum_{i=1}^{n} \partial_{x}^{\alpha} w_{x_{i}} d x,
\end{aligned}
$$

which leads to

$$
e_{j}(w)^{\prime}(t) \leq \frac{\lambda^{\prime}(t)}{\lambda(t)} e_{j}(w)(t)+\sum_{|\alpha|=j}\left\|\partial_{x}^{\alpha} g\right\|_{2}+C_{j} \sum_{k=0}^{j} e_{k}(w)(t) .
$$

Finally we get

$$
E_{N_{0}}(w)^{\prime}(t) \leq\left(\frac{\lambda^{\prime}(t)}{\lambda(t)}+C_{N_{0}}\right) E_{N_{0}}(w)(t)+\|g(t)\|_{H^{N_{0}}}
$$

We can not use Gronwall's Lemma here, since the factor $\frac{\lambda^{\prime}(t)}{\lambda(t)}+C_{N_{0}}$ is unbounded. We apply the Lemma of Nersesyan (Gronwall type lemma for differential inequalities with a singular coefficient) instead:

Lemma 1 (Nersesyan) Let $y(t) \in C([0, T]) \cap C^{1}(0, T)$ be a solution of the differential inequality

$$
y^{\prime}(t) \leq K(t) y(t)+f(t), \quad 0<t<T,
$$

where the functions $K(t)$ and $f(t)$ belong to $C(0, T)$. We assume that for every $t \in(0, T)$ and every $\delta \in(0, t)$

$$
\begin{aligned}
& \int_{0}^{\delta} K(\tau) d \tau=\infty, \quad \int_{\delta}^{T} K(\tau) d \tau<\infty \\
& \lim _{\delta \rightarrow+0} \int_{\delta}^{t} \exp \left(\int_{s}^{t} K(\tau) d \tau\right) f(s) d s \quad \text { exists } \\
& \lim _{\delta \rightarrow+0} y(\delta) \exp \left(\int_{\delta}^{t} K(\tau) d \tau\right)=0 .
\end{aligned}
$$

Then it holds

$$
y(t) \leq \int_{0}^{t} \exp \left(\int_{s}^{t} K(\tau) d \tau\right) f(s) d s .
$$

Using this lemma we obtain with (1.13)

$$
\begin{aligned}
E_{N_{0}}(w)(t) & \leq \int_{0}^{t} \exp \left(\int_{s}^{t} \frac{\lambda^{\prime}(\tau)}{\lambda(\tau)}+C_{N_{0}} d \tau\right)\|g(s)\|_{H^{N_{0}}} d s \\
& \leq C_{g} e^{C_{N_{0}} t} \frac{1}{d-1} \lambda^{d}(t)+C_{g}^{\prime} e^{C_{N_{0}} t} \frac{1}{d} \lambda^{d+1}(t) .
\end{aligned}
$$


During our computations we needed more regularity for $w$ than (1.14). The existence of a solution $w$ with compact support with respect to $x$ and the final estimate (1.17) can be seen as follows: We replace $a(x, t)=\sigma^{2}(x) \lambda^{2}(t)$ by $a_{\varepsilon}(x, t)=\left(\sigma^{2}(x)+\varepsilon\right)(\lambda(t)+\varepsilon)^{2}$ and replace $g$ by a smooth compactly supported function $g_{\varepsilon}$, such that $\left\{g_{\varepsilon}\right\}$ converges to $g$ in the norm of $C\left(\left[0, T_{0}\right], H^{N_{0}}\right)$ if $\varepsilon$ tends to 0 . This modified problem is strictly hyperbolic and has a smooth solution $w_{\varepsilon}$ which is compactly supported and fulfils (1.17). The sequence $\left\{w_{\varepsilon_{k}}\right\}$ $\left(\varepsilon_{k} \rightarrow 0\right)$ converges to a compactly supported solution $w$ of (1.12) satisfying (1.17).

c) We apply this estimate for $w$ to a linearized version of (1.11): Let $\left\{v^{k}\right\}$ be a sequence with

$$
\begin{aligned}
& v^{0}(x, t) \equiv 0, \\
& v_{t t}^{k}-\sum_{i=1}^{n} \partial_{x_{i}}\left(\lambda^{2} \sigma^{2} v_{x_{i}}^{k}\right)=f_{d}\left(x, t, b_{1} v_{x_{1}}^{k-1}, \ldots, b_{n} v_{x_{n}}^{k-1}\right), \\
& v^{k}(x, 0)=v_{t}^{k}(x, 0)=0 .
\end{aligned}
$$

We have $E_{N_{0}}\left(v^{0}\right)(t) \equiv 0 \leq \lambda^{d}(t)$, hence

$$
\left\|f_{d}\left(x, t, b_{1} v_{x_{1}}^{0}, \ldots, b_{n} v_{x_{n}}^{0}\right)\right\|_{H^{N_{0}}} \leq \sqrt{n} C_{f} C_{b} \lambda^{d-1}(t) \lambda^{\prime}(t)+\left(C_{d}^{\prime}+C_{d}\right) \lambda^{d}(t) \lambda^{\prime}(t) .
$$

This implies

$$
E_{N_{0}}\left(v^{1}\right)(t) \leq \sqrt{n} C_{f} C_{b} e^{C_{N_{0}} t} \frac{1}{d-1} \lambda^{d}(t)+\left(C_{d}+C_{d}^{\prime}\right) e^{C_{N_{0}} t} \frac{1}{d} \lambda^{d+1}(t) .
$$

We suppose $\frac{2 C_{f} C_{b} \sqrt{n}}{d-1} \leq \frac{1}{3}$ and restrict the interval for $t$ such that

$$
e^{C_{N_{0}} t} \leq 2, \quad 2\left(C_{d}+C_{d}^{\prime}\right) \frac{1}{d} \lambda(t) \leq \frac{1}{3}
$$

and conclude

$$
E_{N_{0}}\left(v^{1}\right)(t) \leq \frac{2}{3} \lambda^{d}(t) \leq \lambda^{d}(t)
$$

By induction we get the uniform bound $E_{N_{0}}\left(v^{k}\right)(t) \leq \lambda^{d}(t), k \in \mathbb{N}$. In order to show the convergence of the sequence $\left\{v^{k}\right\}$ we consider the sequence of differences $w^{k}=v^{k+1}-v^{k}$. The function $w^{k}$ satisfies

$$
\begin{aligned}
& w_{t t}^{k}-\sum_{i=1}^{n} \partial_{x_{i}}\left(\lambda^{2} \sigma^{2} w_{x_{i}}^{k}\right)=\sum_{i=1}^{n} g_{d k i}(x, t) b_{i}(x, t) w_{x_{i}}^{k-1} \\
& \quad:=f_{d}\left(x, t, b_{1} v_{x_{1}}^{k}, \ldots, b_{n} v_{x_{n}}^{k}\right)-f_{d}\left(x, t, b_{1} v_{x_{1}}^{k-1}, \ldots, b_{n} v_{x_{n}}^{k-1}\right), \\
& w^{k}(x, 0)=w_{t}^{k}(x, 0)=0 .
\end{aligned}
$$

The $L^{2}$-norm of the right-hand side can be bounded by

$$
\sqrt{n} C_{f} C_{b} \frac{\lambda^{\prime}(t)}{\lambda(t)} E_{0}\left(w^{k-1}\right)(t) .
$$


From $E_{0}\left(w^{0}\right)(t) \leq \lambda^{d}(t)$ and the special choice of $d$ we see by induction that

$$
E_{0}\left(w^{k}\right)(t) \leq\left(\frac{2}{3}\right)^{k} \lambda^{d}(t) .
$$

This implies that $\left\{v^{k}\right\}$ is a Cauchy sequence in $C^{1}\left([0, T], L^{2}\right)$. By interpolation and the uniform estimate of $E_{N_{0}}\left(v^{k}\right)(t)$ we deduce that $v^{k}$ converges in $C^{1}\left([0, T], H^{N_{0}-1}\right)$. The limit is a solution of (1.11) and satisfies

$$
\operatorname{supp}(v(., t)) \subset[-M-C t, M+C t] .
$$

This proves the finite propagation speed of the constructed solution. Thus we have shown:

Theorem 1 Let the assumptions (1.4) to (1.10) be satisfied. Then the Cauchy problem (1.3) has a solution $u$ with $u, u_{t}, \sigma u_{x} \in C\left([0, T], H^{N-\gamma}\right)$, where

$$
\gamma=2 d+2, \quad \frac{2 C_{f} C_{b} \sqrt{n}}{d-1} \leq \frac{1}{3} .
$$

This solution has a finite speed of propagation.

Open problems:

1. Uniqueness of the solution in $C^{1}\left([0, T], H^{N-\gamma}\right)$;

2. A local existence result in Gevrey spaces under Gevrey-type Levi conditions.

\subsection{WEAKLY HYPERBOLIC EQUATIONS WITH TIME DEGENERACY AND FAST OSCILLATIONS, BUT WITHOUT SPATIAL DEGENERACY}

In this section we consider the model problem with time degeneracy and oscillations in the coefficients of the main part, but without spatial degeneracy

$$
\begin{aligned}
& u_{t t}-\lambda^{2}(t) a^{2}(t) \triangle u=f\left(b_{1}(x, t) u_{x_{1}}(x, t), \ldots, b_{n}(x, t) u_{x_{n}}(x, t)\right) \\
& u(x, 0)=\varphi_{0}(x), \quad u_{t}(x, 0)=\varphi_{1}(x) .
\end{aligned}
$$

The function $\lambda=\lambda(t)$ describes the degeneracy with respect to $t$ as in the previous section. To understand the influence of oscillations which are produced by the function $a=a(t)$, let us remember a result from [Tar95]. There it was shown that the Cauchy problem for

$$
u_{t t}-\exp \left(-\frac{2}{t^{\alpha}}\right) a^{2}\left(\frac{1}{t}\right) u_{x x}=0
$$

is $C^{\infty}$-well-posed if and only if $\alpha \geq \frac{1}{2}$. Here $a=a(t)$ is a non-constant, 1 -periodic positive function from $C^{\infty}$. This example shows that the product 
between amplitudes and frequencies of the oscillations is important. An interpretation of this example and a definition of so-called fast oscillations by the condition

$$
\left|D_{t} a(t)\right| \leq C \frac{\lambda^{\prime}(t)}{\lambda(t)}|\ln \lambda(t)|, \quad t \in\left(0, T_{0}\right]
$$

were given in [Yag97]. There it was proved that (1.20) is sufficient for $C^{\infty_{-}}$ well-posedness (necessity for (1.19) is clear). We suppose:

- $\lambda=\lambda(t) \in C^{2}\left(\left[0, T_{0}\right]\right)$ satisfies $(1.7)$;

- there exist positive constants $d_{0} \geq \frac{1}{2}$ and $d_{1}$ such that

$$
d_{0} \frac{\lambda(t)}{\Lambda(t)} \leq \frac{\lambda^{\prime}(t)}{\lambda(t)} \leq d_{1} \frac{\lambda(t)}{\Lambda(t)}, \quad\left|\lambda^{\prime \prime}(t)\right| \leq d_{1} \lambda(t)\left(\frac{\lambda(t)}{\Lambda(t)}\right)^{2}
$$

for all $t \in\left(0, T_{0}\right]$, where $\Lambda(t):=\int_{0}^{t} \lambda(\tau) d \tau$;

- the function $a=a(t) \in C^{1}\left(\left(0, T_{0}\right]\right)$ satisfies (1.20) and

$$
0<c \leq a(t) \leq C, \quad t \in\left(0, T_{0}\right] .
$$

Our approach will be divided into the following steps:

- derivation of a strictly hyperbolic type property for the solutions of linear weakly hyperbolic Cauchy problems by using microlocal considerations for explicit solutions of linear ordinary differential equations depending on parameters;

- using the a-priori estimate and a fixed point argument to construct a Sobolev solution of the starting problem (1.18).

a) An essential point to handle quasilinear equations is the strictly hyperbolic type property for corresponding linear equations (compare with step b) of the previous section). If we try to follow the reasoning from the previous section, the conditions (1.20) and (1.22) imply for the energies $E_{N_{0}}$ an inequality of the type (compare with (1.15))

$$
E_{N_{0}}(u)^{\prime}(t) \leq C \frac{\lambda^{\prime}(t)}{\lambda(t)}|\ln \lambda(t)| E_{N_{0}}(u)(t)+\ldots .
$$

Consequently, Lemma 1 is not applicable and it is not clear how to apply the energy method.

To obtain a strictly hyperbolic type property for the Cauchy problem

$$
u_{t t}-\lambda^{2}(t) a^{2}(t) \triangle u=-\lambda^{\prime}(t) f(x, t), \quad u(x, 0)=u_{t}(x, 0)=0
$$

we need another approach, which was developped in [RY97]. One can prove: 
Lemma 2 There exists a Banach space $B_{M, Q, T}$ such that for every $f$ belonging to $B_{M, Q, T}$ one has a uniquely determined solution of (1.23) with the property that $\frac{u}{\lambda}, \frac{u_{t}}{\lambda}$ and $u_{x}$ belong to $B_{M, Q, T}$, too. Moreover, the a-priori estimate

$$
\left\|\frac{u}{\lambda}\right\|_{M, Q, T}+\left\|\frac{u_{t}}{\lambda}\right\|_{M, Q, T}+\left\|\nabla_{x} u\right\|_{M, Q, T} \leq C_{a p r}\|f\|_{M, Q, T}
$$

holds with a constant $C_{a p r}$ independent of $T \in\left(0, T_{0}\right]$ and $f$.

The idea of the construction of $B_{M, Q, T}$ is to describe the elements $u=u(x, t)$ by the behaviour of its partial Fourier transform $\hat{u}=\hat{u}(t, \xi)$ in the cotangent space. Therefore we study the Cauchy problem

$$
D_{t}^{2} \hat{u}-\lambda^{2}(t) a^{2}(t)|\xi|^{2} \hat{u}=\lambda^{\prime} \hat{f}, \quad \hat{u}(0, \xi)=\hat{u}_{t}(0, \xi)=0
$$

under the assumptions $\hat{f} \in C\left([0, T], L^{\infty}\left(\mathbb{R}^{n}\right)\right)$ and $\lambda(t) \in C^{2}\left(\left[0, T_{0}\right]\right),(1.21)$, (1.22).

In the light of (1.24), it is sufficient to show which properties of $\hat{f}$ are transferred to $\frac{\hat{u}}{\lambda}, \frac{\hat{u}_{t}}{\lambda}$ and $\xi_{j} \hat{u}$. This can be done by following the next steps:

- we divide $\mathbb{R}^{n} \times[0, T]$ into the so-called pseudo-differential zone $Z_{p d}$ and the hyperbolic zone $Z_{\text {hyp }}$ ([Yag97]);

- we transform the equation from (1.25) to a first order system in each zone;

- $\quad$ we find a fundamental solution $X=X(t, s, \xi)$ for the first order system in each zone and an estimate of the form ([Yag97])

$$
\|X(t, s, \xi)\| \leq \exp \left(C \int_{s}^{t} g(\tau, \xi) d \tau\right)
$$

- we represent the solution of a suitable Cauchy problem by the aid of the fundamental solution;

- using these explicit representations leads to assumptions for $\hat{f}=\hat{f}(t, \xi)$ of the form

$$
|\hat{f}(t, \xi)| \leq C(\xi) \exp \left(Q \int_{a(\xi)}^{t} g(\tau, \xi) d \tau\right)
$$

where $C(\xi)$ appears because $\xi$ is a parameter and $a(\xi)$ depends on the zone;

- the right-hand sides of these estimates determine the weights in the definition of $B_{M, Q, T}$.

b) Let us go into detail. By $t_{\xi}$ we denote the solution of the equation $\Lambda\left(t_{\xi}\right)\langle\xi\rangle=N \ln \langle\xi\rangle$, where $\langle\xi\rangle=\left(c+|\xi|^{2}\right)^{\frac{1}{2}}$ and $N, c$ are sufficiently large positive numbers. 
The pseudo-differential zone $Z_{p d}(c, N)$ is defined by (see [Yag97])

$$
Z_{p d}(c, N):=\left\{(x, t) \in \mathbb{R}^{n} \times[0, T]: \Lambda(t)\langle\xi\rangle \leq N \ln \langle\xi\rangle\right\} .
$$

Substituting $w_{2}:=D_{t} \hat{u}, w_{1}:=\varrho(t, \xi) \hat{u}$, where $\varrho=\varrho(t, \xi)$ is the positive root of $\varrho^{2}-1-\frac{\lambda^{2}(t)}{\Lambda(t)}\langle\xi\rangle \ln \xi=0,(1.25)$ reads as

$$
\begin{aligned}
& D_{t} W-A(t, \xi) W=F, \quad W(0, \xi)=0, \\
& W=\left(\begin{array}{c}
w_{1} \\
w_{2}
\end{array}\right), \quad F=\left(\begin{array}{c}
0 \\
\lambda^{\prime}(t) \hat{f}
\end{array}\right), \quad A(t, \xi)=\left(\begin{array}{cc}
\frac{D_{t} \varrho}{\varrho} & \varrho \\
\lambda^{2}(t) a^{2}(t)|\xi|^{2} & 0
\end{array}\right) .
\end{aligned}
$$

If $X=X(t, s, \xi)$ is the fundamental matrix, that is, it solves

$$
D_{t} X-A(t, \xi) X=0, \quad X(s, s, \xi)=I, \quad 0 \leq s \leq t \leq t_{\xi},
$$

then $W(t, \xi)=\int_{0}^{t} X(t, s, \xi) F(s, \xi) d \xi$ solves $(1.26)$.

The assumptions concerning $\lambda, a$ and the definition of $Z_{p d}(c, N)$ imply $\|A(t, \xi)\| \leq C_{p d} g(t, \xi)$, where $g(t, \xi)=\varrho(t, \xi)+\frac{\varrho_{t}(t, \xi)}{\varrho(t, \xi)}$. Consequently, using the matrizant we obtain

$$
\|X(t, s, \xi)\| \leq \exp \left(\int_{s}^{t}\|A(\tau, \xi)\| d \tau\right) \leq \exp \left(C_{p d} \int_{s}^{t} g(\tau, \xi) d \tau\right) .
$$

Thus, on the one hand, we have the representations

$$
\begin{aligned}
\varrho(t, \xi) \hat{u}(t, \xi) & =\int_{0}^{t} X_{12}(t, s, \xi) \lambda^{\prime}(s) \hat{f}(s, \xi) d s, \\
D_{t} \hat{u}(t, \xi) & =\int_{0}^{t} X_{22}(t, s, \xi) \lambda^{\prime}(s) \hat{f}(s, \xi) d s,
\end{aligned}
$$

on the other hand it is clear how to estimate $\left|X_{12}(t, s, \xi)\right|,\left|X_{22}(t, s, \xi)\right|$. Both results together yield a strictly hyperbolic type result for the solution of (1.25) in $Z_{p d}(c, N)$.

Lemma 3 If $\hat{f}(t, \xi) \in C\left([0, T], L^{\infty}\left(\mathbb{R}^{n}\right)\right)$ satisfies the inequality

$$
|\hat{f}(t, \xi)| \leq A(\xi) \exp \left(Q \int_{t_{\xi}}^{t} g(\tau, \xi) d \tau\right), \quad Q>C_{p d}
$$

in $Z_{p d}(c, N)$, then the solution $\hat{u}(t, \xi)$ of $(1.25)$ satisfies in $Z_{p d}(c, N)$ the estimates

$$
\begin{aligned}
& \left|\xi_{j} \hat{u}(t, \xi)\right| \leq C \frac{A(\xi)}{Q-C_{p d}} \exp \left(Q \int_{t_{\xi}}^{t} g(\tau, \xi) d \tau\right), \\
& \left|\hat{u}_{t}(t, \xi)\right| \leq C A(\xi) \lambda(t) \exp \left(Q \int_{t_{\xi}}^{t} g(\tau, \xi) d \tau\right) .
\end{aligned}
$$


Remark 1 The Cauchy problem (1.25) feels neither oscillations nor hyperbolicity in $Z_{p d}(c, N)$.

c) Now let us devote to the hyperbolic zone ([Yag97]):

$$
Z_{\text {hyp }}(c, N):=\left\{(\xi, t) \in \mathbb{R}^{n} \times[0, T]: \Lambda(t)\langle\xi\rangle \geq N \ln \langle\xi\rangle\right\} .
$$

Let us denote the characteristic roots by

$$
\tau_{i}(t, \xi):=(-1)^{i} \lambda(t) a(t)|\xi| .
$$

There exists a well-known approach for the construction of fundamental solutions in the strictly hyperbolic case. One knows how to apply the perfect diagonalizer. It is not clear how to use this tool for equations with fast oscillations. Nevertheless in [Yag97] it was done only the first step of perfect diagonalization to derive $C^{\infty}$-well-posedness for linear weakly hyperbolic equations with fast oscillations. One can use this idea to derive a corresponding result to Lemma 1.19 in $Z_{\text {hyp }}(c, N)$. Using suitable transformations, diagonalization and one step of perfect diagonalization transforms (1.25) to

$$
D_{t} W-\left(\begin{array}{cc}
\tau_{1} & 0 \\
0 & \tau_{2}
\end{array}\right) W+F W+B W=N^{-1} \frac{\lambda^{\prime}(t) \hat{f}}{2 a(t)}\left(\begin{array}{c}
-1 \\
1
\end{array}\right)
$$

in $Z_{\text {hyp }}(c, N)$, where $F:=\frac{1}{2} \frac{D_{t} a(t)}{a(t)} I, N$ is the diagonalizer and $B$ satisfies

$$
\|B(t, \xi)\| \leq C\left(\frac{\lambda(t)}{\Lambda(t)}+\frac{\lambda(t)|\ln \lambda(t)|^{2}}{\langle\xi\rangle \Lambda^{2}(t)}\right)=: K(t, \xi),
$$

see [Yag97]. If $X(t, s, \xi)$ is a fundamental solution of $(1.27)$, then

$$
W(t, \xi)=\int_{t_{\xi}}^{t} X(t, s, \xi) F(s, \xi) d s+X\left(t, t_{\xi}, \xi\right) W\left(t_{\xi}, \xi\right)
$$

solves (1.27) with a Cauchy condition on $t=t_{\xi}$. Using the ansatz $X(t, s, \xi)=$ $E(t, s, \xi) Q(t, s, \xi)$, where $E$ is the fundamental matrix of the left-hand side of (1.27) without $B W$, the norms of $Q(t, s, \xi), X(t, s, \xi)$, respectively, can be estimated by $C \exp \left(C_{h y p} \int_{s}^{t} K(\tau, \xi) d \tau\right), t_{\xi} \leq s \leq t$. This is exactly what we need to prove a strictly hyperbolic type estimate in $Z_{\text {hyp }}(c, N)$.

Lemma 4 . If $\hat{f} \in C\left([0, T], L^{\infty}\left(\mathbb{R}^{n}\right)\right)$ satisfies the inequality

$$
|\hat{f}(t, \xi)| \leq C\langle\xi\rangle^{-M} \exp \left(Q \int_{T_{0}}^{t} K(\tau, \xi) d \tau\right), \quad Q>C_{h y p}
$$

in $Z_{\text {hyp }}(c, N)$, then

$$
\begin{aligned}
& \left|\xi_{j} \hat{u}(t, \xi)\right| \leq C\langle\xi\rangle^{-M} \exp \left(Q \int_{T_{0}}^{t} K(\tau, \xi) d \tau\right), \\
& \left|\hat{u}_{t}(t, \xi)\right| \leq C\langle\xi\rangle^{-M} \lambda(t) \exp \left(Q \int_{T_{0}}^{t} K(\tau, \xi) d \tau\right) .
\end{aligned}
$$


Remark 2 In opposite to $Z_{p d}(c, N)$ the Cauchy problem (1.25) feels oscillations as well as hyperbolicity in $Z_{\text {hyp }}(c, N)$.

d) Now we are able to construct $B_{M, Q, T}$. Therefore we introduce the weight function $\left((\xi, t) \in \mathbb{R}^{n} \times[0, T]\right)$

$$
\mathcal{N}_{Q, M}(t, \xi):= \begin{cases}\langle\xi\rangle^{M} \exp \left(Q \int_{t}^{T_{0}} K(\tau, \xi) d \tau\right) & : t \in\left[t_{\xi}, T_{0}\right], \\ \langle\xi\rangle^{M} \exp \left(Q \int_{t_{\xi}}^{T_{0}} K(\tau, \xi) d \tau+Q \int_{t}^{t_{\xi}} g(\tau, \xi) d \tau\right) & : t \in\left[0, t_{\xi}\right] .\end{cases}
$$

For a given positive number $M$ we denote by $B^{M}$ the normed linear space

$$
B^{M}=\left\{u \in \mathcal{S}^{\prime}\left(\mathbb{R}^{n}\right): \hat{u} \in L_{l o c}^{\infty} \text { is a function with } \sup _{\xi \in \mathbb{R}^{n}}\langle\xi\rangle^{M}|\hat{u}(\xi)|<\infty\right\} .
$$

For given positive numbers $M, Q$ and $T, T \leq T_{0}$, we denote by $B_{M, Q, T}$ the linear space

$$
\begin{aligned}
& B_{M, Q, T}=\left\{u \in C\left([0, T], B^{M}\right): \hat{u}(\xi, t) \text { satisfies } \mathcal{N}_{Q, M}(t, \xi)|\hat{u}(\xi, t)| \leq C\right. \\
&\left.\forall(\xi, t) \in \mathbb{R}^{n} \times[0, T]\right\} .
\end{aligned}
$$

One can show that $B_{M, Q, T}$ is a Banach space with the norm

$$
\|u\|_{M, Q, T}:=\max _{[0, T]} \sup _{\xi \in \mathbb{R}^{n}} \mathcal{N}_{Q, M}(t, \xi)|\hat{u}(\xi, t)| .
$$

All results of this section together make it possible to prove Lemma 2 .

e) In the last point let us study (1.18) by the aid of the spaces $B_{M, Q, T}$.

- To have a reasonable space for the data we can use the imbedding $C\left([0, T], W_{1}^{r}\left(\mathbb{R}^{n}\right)\right) \subset B_{M, Q, T}$ if $r \geq r_{0}$, where $r_{0}$ depends on the given $M$ and $Q$.

- To have a reasonable space for the solution we can use the imbedding $B_{M, Q, T} \subset C\left([0, T], H^{l}\left(\mathbb{R}^{n}\right)\right)$ if $M-l>\frac{n}{2}, l>0$ and $Q>0$.

- Up to now we are not able to study superposition operators in $B_{M, Q, T}$.

- The only way for us to include nonlinearities is to prove that $B_{M, Q, T}$ is even a Banach algebra (see [RY97]).

- Thus one can suppose that $f$ depends on $b_{i}(x, t) u_{x_{i}}(x, t)$ analytically. To simplify the calculations in [RY97] we restricted ourselves to the assumption that $f$ be entire.

Now we can use the approach of the previous section to construct a uniquely determined solution of (1.18). We do not need a reduction process, we only use successive approximation and the a-priori estimate (1.24). To obtain a Cauchy sequence in a suitable space $B_{M, Q, T}$ we have to suppose a Levi condition which 
is close to the sharp Levi condition $O\left(\lambda^{\prime}(t)\right)$. Let us formulate a final result, where we renounce to represent the most general version.

Theorem 2 Let us consider the Cauchy problem

$$
\begin{aligned}
& u_{t t}-\lambda^{2}(t) a^{2}(t) \triangle u=f\left(b_{1}(x, t) u_{x_{1}}(x, t), \ldots, b_{n}(x, t) u_{x_{n}}(x, t)\right), \\
& u(x, 0)=\varphi_{0}(x), \quad u_{t}(x, 0)=\varphi_{1}(x)
\end{aligned}
$$

under the assumptions $\lambda \in C^{2}([0, T]),(1.7),(1.21)$ and (1.22) and the assumptions for $f$

- $f$ is polynomial in its arguments,

- $\quad b_{k}=b_{k}(x, t) \in C\left(\left[0, T_{0}\right], W_{1}^{s}\left(\mathbb{R}^{n}\right)\right)$ for every given $s \in \mathbb{N}$, where

$$
\left\|b_{k}(., t)\right\|_{W_{1}^{s}\left(\mathbb{R}^{n}\right)} \leq o\left(\lambda^{\prime}(t)\right), \quad t \in\left(0, T_{0}\right], \text { (Levi condition). }
$$

Then there exists a (in general sufficiently large) constant $r$ such that for arbitrary data $\varphi_{0}, \varphi_{1}$ belonging to $W_{1}^{r}\left(\mathbb{R}^{n}\right), W_{1}^{r-1}\left(\mathbb{R}^{n}\right)$, respectively, we have a locally defined solution

$$
u \in C\left([0, T], H^{\left[\frac{n}{2}\right]+3}\left(\mathbb{R}^{n}\right)\right) \cap C^{1}\left([0, T], H^{\left[\frac{n}{2}\right]+2}\left(\mathbb{R}^{n}\right)\right) \cap C^{2}\left([0, T], H^{\left[\frac{n}{2}\right]+1}\left(\mathbb{R}^{n}\right)\right) .
$$

The solution is uniquely determined in $B_{M, Q, T}$.

Open problems:

1. Is it possible to apply the energy method to (1.18) under the assumptions of this section?

2. Find a strictly hyperbolic type result for the Cauchy problems for

$$
u_{t t}-\lambda^{2}(t) a^{2}(t) \triangle u+\sum_{i=1}^{n} b_{i}(x, t) u_{x_{i}}=f
$$

under the Levi condition

$$
\left\|b_{i}(x, t)\right\|_{W_{1}^{s}\left(\mathbb{R}^{n}\right)} \leq O\left(\lambda^{\prime}(t)\right), \quad t \in\left(0, T_{0}\right]
$$

and

$$
u_{t t}-\lambda^{2}(t) a^{2}(t) \sigma^{2}(x) \triangle u=f
$$

Remark 3 We omit a long list of references. But each interested reader can have a list of publications concerning the subject of this paper after contacting one of the authors. 


\section{References}

[CJS83] Colombini, F., Jannelli, E., and Spagnolo, S. Well-posedness in the Gevrey classes of the Cauchy problem for a non-strictly hyperbolic equation with coefficients depending on time. Ann. Sc. Norm. Sup. Pisa, 10:291-312, 1983.

[CL95] Colombini, F. and Lerner, N. Hyperbolic operators with non-Lipschitz coefficients. Duke Math. J., 77:657-698, 1995.

[CS82] Colombini, F. and Spagnolo, S. An example of a weakly hyperbolic Cauchy problem not well posed in $C^{\infty}$. Acta Math., 148:243-253, 1982.

[D'A95] D'Ancona, P. A note on a theorem of Jörgens. Math. Z., 218:239-252, 1995.

[IP74] Ivrii, V.Ya. and Petkov, V.M. Necessary conditions for the wellposedness of the Cauchy problem for non-strictly hyperbolic equations (in Russian). Usp. Mat. Nauk, 29(5):3-70, 1974. English translation: Russ. Math. Surv. 29(5):1-70, 1974.

[Ole70] Oleinik, O.A. On the Cauchy problem for weakly hyperbolic equations. Comm. pure appl. math., 23:569-586, 1970.

[Qi 58] Qi Min-you. On the Cauchy problem for a class of hyperbolic equations with initial data on the parabolic degenerating line. Acta Math. Sinica, 8:521-529, 1958.

[RY97] Reissig, M. and Yagdjian, K. Weakly hyperbolic equations with fast oscillating coefficients. Preprint 7, Technische Universität Bergakademie Freiberg, 1997.

[Tar95] Tarama, S. On the second order hyperbolic equations degenerating in the infinite order. Math. Japonica, 42:523-534, 1995.

[Yag97] Yagdjian, K. The Cauchy Problem for Hyperbolic Operators. Multiple Characteristics, Micro-Local Approach. Akademie Verlag, Berlin, 1997. 